\title{
EL RETORNO DE MOSCÚ: LA GRAN ESTRATEGIA DE RUSIA EN LA ERA PUTIN (2000-2020) ${ }^{1}$
}

\author{
THE RETURN OF MOSCOW: RUSSIA'S GRAND \\ STRATEGY IN THE PUTIN ERA (2000-2020)
}

\author{
LE RETOUR DE MOSCOU: LA GRANDE STRATÉGIE \\ RUSSE À L'ÈRE POUTINE (2000-2020)
}

\author{
Ariel GonzÁlez Levaggi \\ Pontificia Universidad Católica Argentina \\ agonzalez@uca.edu.ar
}

REsumEN: Desde el ascenso de Vladimir Putin al poder, Rusia ha combinado
estabilidad política y económica con asertividad internacional, lo que ha
sentado las bases para el regreso de Moscú a la primera plana del escenario
mundial. El artículo presenta las principales discusiones en torno a la gran
estrategia de la Federación de Rusia y analiza la trayectoria de su política ex-
terior desde la llegada de Vladimir Putin al poder hasta el inicio de la crisis
global de la covid-19. Desde la perspectiva del realismo neoclásico, el princi-
pal argumento es que Rusia posee una gran estrategia pragmática basada en
un trípode de objetivos -preservación de estatus como potencia global, pri-
macía en el espacio postsoviético y disuasión a la expansión de la oTAN- que
se implementan mediante la utilización de una gama amplia de herramien-
tas, incluida la coerción por vías militares. A continuación, se desarrolla la
evolución de la estrategia internacional del pragmatismo estratégico ruso que
generó una serie de tensiones con Occidente tras las crisis de Georgia en

${ }^{1} \mathrm{El}$ artículo se desprende del proyecto de investigación "Estrategias marítimas de las potencias emergentes (República Popular de China, Federación de Rusia y la República de la India) y los océanos del sur global”, financiado por la Sede de Investigación y Estudios Estratégicos Navales (sIEN) de la Armada Argentina. El autor agradece al contraalmirante (R) Ricardo Alessandrini y al contraalmirante (R) Eduardo Castro Rivas por el apoyo a la investigación. Además agradece a Silvana Elizondo, CN (R) Jorge Defensa, Paulo Botta y Daiana D'Elia por sus comentarios y recomendaciones que enriquecieron el trabajo. 
2008 y Ucrania en 2014, en paralelo con el ascenso de un vector alternativo eurasiático.

Palabras clave: Rusia; Putin; gran estrategia; pragmatismo estratégico; Occidente; Eurasia.

Abstract: Since Vladimir Putin's ascent to power, Russia has combined political and economic stability with a greater assertiveness on the global stage, which has laid the groundwork for Moscow's return to the forefront of international politics. The paper presents the principal discussions around the grand strategy of the Russian Federation and analyzes the trajectory of its foreign policy from the arrival of Vladimir Putin to power to the beginning of the global covid-19 crisis. From the perspective of neoclassical realism, the principal argument is that Russia is implementing a pragmatic strategy based on a trio of objectives: preservation of its status as a global power, maintaining its hegemonic role in the post-Soviet realm, and preventing the expansion of NATO. These are implemented using a wide range of tools, including military coercion. The paper then addresses the evolution of Russia's pragmatic international strategy, which has led to a series of tensions with the West following the crisis in Georgia in 2008 and in Ukraine in 2014, in parallel with the ascent of an alternative Euro-Asian vector.

Keywords: Russia; Putin; grand strategy; strategic pragmatism; West; Eurasia.

\section{Traducción de Gonzalo Celorio Morayta}

Résumé: Depuis l'arrivée de Vladimir Poutine au pouvoir, la Russie a su conjuguer stabilité politico-économique et fermeté au niveau international, jetant ainsi les bases de son retour sur le devant de la scène mondiale. Cet article présente les principaux débats ayant trait à la grande stratégie de la Fédération de Russie, avant d'analyser l'évolution de sa politique étrangère, de l'arrivée au pouvoir de Vladimir Poutine au début de la pandémie de COVID-19. Le réalisme néoclassique centre son approche sur ce qui serait la grande stratégie pragmatique russe reposant sur trois objectifs - maintien du statut de puissance mondiale de la Russie, hégémonie au sein de l'espace postsoviétique et contention de l'OTAN, poursuivis à travers l'emploi d'un large éventail de moyens qui incluent la coercition militaire. Cet article expose l'évolution de la stratégie internationale propre au pragmatisme stratégique russe, à l'origine d'une série de 
tensions avec l'Occident suite aux crises géorgienne de 2008 et ukrainienne de 2014, et ceci parallèlement à l'essor d'un axe alternatif eurasiatique.

Mots clés: Russie; Poutine; grande stratégie; pragmatique stratégique;

Occident; Eurasie.

\section{Traducción de ARIEL ElBAZ}

Fecha de recepción: octubre de 2019

Fecha de aceptación: junio de 2020

$\mathrm{H}$ acia fines de 1991, la Federación de Rusia nace de entre las cenizas de la Unión de las Repúblicas Socialistas Soviéticas (URSs), heredando tanto sus glorias pasadas como el fracaso del modelo político y económico comunista. Uno de los elementos críticos del fin de la Guerra Fría fue la desaparición de Rusia como un polo del sistema internacional. Cuando la Federación de Rusia se establece como una entidad soberana el 25 de diciembre de 1991, había perdido el $23.8 \%$ de los territorios, casi la mitad de la población y el $41 \%$ del pib que correspondían a la uRss. Moscú sufrió una pérdida de su peso relativo en el orden internacional, además del desgaste progresivo de las capacidades estatales en múltiples planos. Además, la decadencia se profundizó durante los años noventa. En el periodo 1992-1999, su PIB descendió casi $50 \%$ mientras el gasto militar se retrajo abruptamente de 48.6 a 18000 millones de dólares. A pesar de sostener relaciones generalmente amigables con EEUU y los principales países europeos, Rusia atravesó un ciclo vicioso de crisis y decadencia.

Con la llegada de Putin al poder en el año 2000 y luego de dos décadas de estabilidad política y económica, la Federación de Rusia recuperó parte de su relevancia en el sistema internacional. Hacia 2018, la economía se encontraba en el puesto 11 medido en términos nominales y en el puesto $6^{\text {to }}$ a valores de paridad de poder adquisitivo (PPA) del produc- 
to interno bruto (PIB), según datos del Fondo Monetario Internacional (FMI). Además de mantener grandes cantidades de material estratégico -fundamentalmente misiles intercontinentales y el principal arsenal de armas nucleares a nivel global-, el gasto militar saltó de 9 a 66000 millones de dólares en el periodo 2000-2018 aunque en el periodo 2013-2018 disminuyó más de 25\% en dólares corrientes, de acuerdo con el relevamiento de gastos militares del Instituto Internacional de Estudios para la Paz de Estocolmo (SIPRI). Desde 2018, Rusia ha quedado fuera del top cinco a pesar de gastar anualmente alrededor de $3.9 \%$ de su PIB en defensa, aunque esta medición no toma en consideración otros gastos que agregarían más de un punto porcentual de PIB adicional. ${ }^{2}$

A lo largo de la era Putin, Rusia ha recuperado su rol en el actual orden internacional, con luces y sombras. Si bien la Federación es una potencia mediana en lo económico y su presupuesto militar no se encuentra a la par de su pasado soviético, ha sido lo suficientemente selectiva para que sus limitados activos logren resultados eficientes, como fue el caso de la neutralización de Georgia como un proxy atlantista en el Cáucaso, la recuperación de la península de Crimea en el Mar Negro y la intervención de ultramar en la Guerra Civil en Siria. En la primera sección del artículo se analizan los principales elementos de la estrategia pragmática en la era Putin, para luego desarrollar la evolución temporal de la agenda internacional rusa centrada en las complejas relaciones con Occidente y el fortalecimiento de su dimensión euroasiática.

${ }^{2}$ Michael Kofman, "Russian defense spending is much larger, and more sustainable than it seems", Defense News, 2019, https://www.defensenews.com/opinion/commentary/2019/05/03/russian-defense-spending-is-much-larger-and-more-sustainable-than-it-seems / 
Pragmatismo estratégico: la gran estrategia de Putin

A pesar del nuevo activismo ruso en los asuntos internacionales, las discusiones sobre la gran estrategia después de la Guerra Fría han sido escasas. Una gran estrategia debe centrarse en la política entendida como la conjugación de los elementos militares y no militares para la preservación y proyección de los intereses a largo plazo. ${ }^{3}$ En este sentido, el artículo se enmarca en la escuela del realismo neoclásico que centra la explicación de la conducta externa de los Estados teniendo en cuenta tanto la distribución del poder en el sistema internacional como el rol de las percepciones, capacidades y operatividad del nodo ejecutivo para implementar dicha política. ${ }^{4}$ Taliaferro, Ripsman y Lobell -principales referentes del realismo neoclásico- definen la estrategia internacional como un "principio ordenador que anima todas las relaciones de un Estado con el mundo exterior, con el fin de asegurarse y maximizar sus intereses". ${ }^{5}$ Con base en esta última definición, destacamos tres elementos de una gran estrategia, su principio ordenador, los objetivos y las herramientas utilizadas. En el caso de la Federación de Rusia en la era Putin, el argumento principal es que la gran estrategia pragmática de Moscú está basada en un trípode de objetivos permanentes - preservación de estatus como potencia global, primacía en el espacio postsoviético y disuasión a la expansión de la OTAN- que se combinan con la utilización de una gama amplia de herramientas, incluida la coerción militar.

${ }^{3}$ Paul Kennedy, "Grand Strategy in War and Peace: Toward a Broader Definition”, en Paul Kennedy (ed.), Grand Strategies in War and Peace, New Haven, Yale University Press, 1991, p. 5.

${ }^{4}$ Víctor M. Mijares, "Realismo neoclásico: ¿El retorno de los estudios internacionales a la ciencia política?", Revista de Ciencia Política, 35, 3 (2015), p. 582.

${ }^{5}$ Jeffrey Taliaferro; Norrin Ripsman y Steven Lobell, "Introduction: Grand Strategy between the World Wars", en Jeffrey Taliaferro, Norrin Ripsman y Steven Lobell (eds.), The Challenge of Grand Strategy: The Great Powers and the Broken Balance between the World Wars, Nueva York, Cambridge, University Press, 2012, p. 15. 


\section{El pragmatismo como principio ordenador}

Una de las principales discusiones sobre la gran estrategia en la era Putin se centra justamente en su naturaleza. Tsygankov divide las perspectivas de los analistas sobre la estrategia internacional rusa entre escépticos y alarmistas. ${ }^{6}$ Para los primeros, la actual administración del Kremlin carece de un proyecto estratégico debido a factores internos que impiden el desarrollo de políticas de Estado, como la informalidad del "sistema", la disputa entre líderes de distintos bloques y la arbitrariedad política. ${ }^{7}$ Desde esta perspectiva, se percibe a Rusia como un actor que continúa debilitado por la "competencia de los clanes rivales dentro del Kremlin y la clase política en general". ${ }^{8}$ Rusia se comporta como un actor táctico y oportunista que no tiene una guía clara de acción y desarrolla entonces una "estrategia menos que grande". 9

Por el contrario, los alarmistas enfatizan la existencia de una gran estrategia en clave de amenaza antioccidental y caracterizan a Putin como un líder que cuenta con un "plan maestro" que busca la restauración de la influencia global mediante la división de Occidente. ${ }^{10}$ En esa línea, Rusia tiene una creciente capacidad de "formular una gran estrategia coherente, pero tal estrategia es antidemocrática y antioccidental en sus principales orientaciones". ${ }^{11}$ En general, esta perspectiva tiene arraigo en un sector importante de la aca-

${ }^{6}$ Andrei Tsygankov, "Preserving Influence in a Changing World: Russia's Grand Strategy", Problems of Post-Communism, 58, 2 (2011): pp. 29-30.

7 Andrew Monaghan, "Defibrillating the Vertikal? Putin and Russian Grand Strategy", Research Paper, Londres, Chatham House, 2014, pp. 2-3.

${ }^{8}$ Tsygankov, art. cit., p. 29.

${ }^{9}$ Celeste Wallander, "Russia: The Domestic Sources of a Less-than-aGrand Strategy", en Ashley J. Tellis y Michael Wills (eds.), Strategic Asia 2007-2008: Domestic Political Change and Grand Strategy, Seattle, National Bureau of Asian Research. 2007.

${ }^{10}$ Douglas Schoen y Evan Smith, Putin's Master Plan: To Destroy Europe, Divide NATO, and Restore Russian Power and Global Influence, Nueva York, Encounter Books, 2016.

${ }^{11}$ Tsygankov, art. cit., p. 30. 
demia y think tanks conservadores en Occidente, ${ }^{12}$ además de ser uno de elementos rescatados por la narrativa de la "Nueva Guerra Fría". ${ }^{13}$ Sin embargo, este tipo de interpretaciones hacen perder la capacidad de desarrollar otro tipo de estrategias generales con una aproximación menos ofensiva. De acuerdo con Kofman, el liderazgo ruso puede no tener algo que satisfaga a la comunidad académica de Occidente, como una gran estrategia en el sentido clásico, pero sí posee una perspectiva estratégica. ${ }^{14}$

En este sentido, Moscú sostiene una serie de lineamientos que bien pueden considerarse como parte de una estra-

12 En la actual visión oficial rusa expresada en sus documentos Concepto de Política Exterior (2016) y la Estrategia de Seguridad Nacional (2015), Occidente se define geográficamente por la región euroatlántica que incluye a los miembros de la Organización del Tratado del Atlántico Norte (OTAN) y la Unión Europea (UE). En este contexto, se subraya el concepto de 'potencias occidentales tradicionales', identificando a EEUU y sus aliados como el nodo principal. Uno de los debates académicos más ricos sobre la identidad rusa se refieren a las cambiantes definiciones de la relación de Rusia con Occidente tanto en términos civilizacionales como político, económicos e internacionales. Véanse, por ejemplo, Iver Neumann, Russia and the Idea of Europe. A Study in Identity and International Relations, Nueva York, Routledge, 1996; Andrey Tsygankov, Russia and the West from Alexander to Putin: Honor in international relations, Cambridge, University Press, 2012.

${ }^{13}$ Entre los principales referentes de esta tesis se encuentran Edward Lucas, The New Cold War: The Future of Russia and the Threat to the West, Nueva York, Palgrave Macmillan, 2008; Robert Legvold, Return to Cold War, Londres, Polity, 2016; Stephen Cohen, Why Cold War Again? How America Lost Post-Soviet Russia, Londres, I.B. Tauris, 2017. Diversos autores no coinciden con esta denominación y entre otros conceptos proponen caracterizar las relaciones ruso-estadounidenses como de 'paz fría', como Richard Sakwa, Frontline Ukraine: Crisis in the Borderlands, Londres, I.B., Tauris, 2015, p. 5, o 'paz caliente', Michael McFaul, From Cold War to Hot Peace. An American Ambassador in Putin's Russia, Nueva York, Houghton Mifflin Harcourt, 2018.

${ }^{14}$ Michael Kofman, "Raiding and International Brigandry: Russia's Strategy for Great Power Competition", War on the Rocks, 2018, https:/ / warontherocks.com/2018/06/raiding-and-international-brigandry-russias-strategy-for-great-power-competition/ 
tegia general. En los ámbitos especializados de discusión se presenta una disyuntiva entre aquellos que perciben Moscú como un actor con una disposición ya sea defensiva u ofensiva, centrado particularmente en su relación con Occidente. Para aquellos que suscriben la primera aproximación, entienden que la política del Kremlin se encuentra "enfocada en asegurar fronteras geográficas, mejorar las condiciones políticas y económicas, y ganar reconocimiento internacional como una potencia con una voz relevante en los asuntos internacionales", ${ }^{15}$ mientras que para la explicación alternativa existe un "impulso expansionista en el que Vladimir Putin busca restaurar la grandeza rusa y revisar las premisas de la seguridad europea". ${ }^{16}$ Una tercera opinión -la cual suscribe el presente artículo- sostiene una aproximación intermedia o mixta, dada por la implementación de prácticas pragmáticas con una serie de objetivos claramente definidos. ${ }^{17}$

En términos generales, la estrategia internacional rusa tiene un grado de coherencia relativamente alto que se refleja en sus principales documentos oficiales. Esto se suma a declaraciones y conceptos clave vertidos por los principales referentes del Kremlin, de manera particular por el presidente Vladimir Putin y el primer ministro Dimitri Medvédev (2012-2020). El diseño y la implementación de una estrategia como ésta requiere consensos previos implementados por un liderazgo político. En este sentido, es importante subrayar dos elementos. Por un lado, la fuerte centralización de la toma de decisiones del régimen autoritario competitivo centrado en la figura de Vladimir Putin. Por el otro, la capacidad del sistema burocrático de establecer consensos o puntos de convergencia expresados en documentos oficiales.

En relación con el sistema centralizado, el sistema 'putinista’ modificó el esquema institucional ruso. Formalmente, la

15 Tsygankov, art. cit., p. 30.

${ }^{16}$ Monaghan, art. cit. p. 3.

17 Dmitri Trenin, "Vladimir Putin's Fourth Vector", Russia in Global Affairs, núm. 1 (enero-marzo de 2018), https://eng.globalaffairs.ru/number/Vladimir-Putins-Fourth-Vector-19441 
Constitución de 1993 estableció una clara división de poderes pese al énfasis colocado en la figura presidencial que nombra -con acuerdo parlamentario- al primer ministro, preside el Consejo de Seguridad, tiene la capacidad de disolver la Duma y ejerce la dirección de la política exterior, además de ser el jefe supremo de las Fuerzas Armadas con la capacidad de declarar el estado de guerra. Las reformas constitucionales propuestas en el discurso anual sobre el estado de la nación a principios de 2020 no modifican esencialmente el sistema formal, más allá de proponer mayores prerrogativas al Consejo de Estado y la Duma estatal. En el periodo 2000-2020, la posición de Vladimir Putin como presidente (2000-2008 y 2012-a la fecha) o como primer ministro (2008-2012) ha representado el ápice del poder ruso postsoviético, aunque no tanto como un líder absoluto, sino como un árbitro entre diferentes grupos de interés dentro del Kremlin.

Sin embargo, las posiciones formales de los principales actores son tan importantes como la estructuración de redes de confianza en el esquema oficial. En la era Putin se desarrolló un 'estado dual' en el cual conviven dos tipos de sistemas: el formal constitucional con uno informal prerrogativo, regido por un régimen administrativo que opera doblemente como "una red de relaciones sociales en la que poderes políticos y económicos se entrelazan en el panorama cambiante de la política fraccional y un actor en el proceso político". ${ }^{18}$ En dicho esquema, el bloque de los siloviki -actores vinculados históricamente a los servicios de seguridad interna- y las empresas estatales tienen un peso fundamental en las decisiones relacionadas con las cuestiones relativas a la política exterior y de seguridad nacional. ${ }^{19}$

18 Richard Sakwa, "Dualism at Home and Abroad: Russian Foreign Policy NeoRevisionism and Bicontinentalism”, en David Cadier y Margot Light (eds.), Russia's Foreign Policy, Londres, Palgrave Macmillan, 2015 , p. 66.

${ }^{19}$ Evgeny Minchenko, "Politburo 2.0: Renovation instead of dismantling”, Moscú, Minchenko Consulting Communications Group, 2017. 
El trípode: estatus global, primacía regional y disuasión a la OTAN

Una serie de documentos oficiales expresan una importante coordinación interagencial entre las principales agencias de análisis, diseño e implementación de la estrategia rusa. ${ }^{20}$ Estos documentos presentan tanto las percepciones sobre el entorno externo en un momento particular como los objetivos, las jerarquías de prioridades y las líneas de acción en sentido general. Entre los principales documentos se destacan el "Concepto de política exterior de la Federación de Rusia” (última versión de 2016), "La estrategia de seguridad nacional de la Federación de Rusia" (2015), la "Doctrina militar de la Federación de Rusia" (2014) y la "Doctrina marítima de la Federación de Rusia" (2015).

En este sentido, el planteamiento de los principales desafíos refleja un esfuerzo de equilibrio interno como el desarrollo de capacidades estatales tradicionales y no tradicionales, así como para limitar los condicionantes externos. Hay una serie de elementos que son recurrentes entre los documentos, como la existencia de un mundo policéntrico, la oposición desde EEUU y sus aliados occidentales a los intereses rusos, la importancia de rol del factor militar en la política mundial, la creciente multiplicación de conflictos regionales y los desafíos que presenta la expansión de la infraestructura militar de la OTAN.

Como afirma la última Estrategia de Seguridad Nacional Rusa,

El fortalecimiento de Rusia se lleva a cabo en un contexto de nuevas amenazas para la seguridad nacional que son de naturaleza múltiple e interconectada. La implementación por la Federación de Rusia de una política exterior y doméstica inde-

${ }^{20}$ En la confección de estos documentos participan desde el Consejo de Seguridad Nacional hasta las corporaciones estatales vinculadas con asuntos estratégicos como Rostec (industria de defensa) o Rosatom (industria nuclear), pasando por ministerios clave como Asuntos Exteriores, Defensa y Desarrollo del Extremo Oriente ruso y el Ártico, entre otros. 
pendiente está dando lugar a la oposición de EEUU y sus aliados, que buscan mantener su dominio en los asuntos mundiales. La política de contención a Rusia que están implementando contempla el ejercicio de presiones políticas, económicas, militares e informativas sobre ella. ${ }^{21}$

En el caso de los intereses nacionales rusos, el concepto de política exterior de la Federación de Rusia identifica primariamente la consecución de la seguridad nacional, soberanía e integridad territorial; la creación de un ambiente externo favorable para propiciar la modernización y competitividad de la economía así como la mejor calidad de vida de su población; la consolidación de la Federación como un centro de influencia global, el fortalecimiento de la posición económica rusa, la promoción de la paz y la seguridad internacionales, la consecución de relaciones amistosas con los países adyacentes y la protección de las minorías rusas en el extranjero, entre otros. ${ }^{22}$

Los intereses nacionales se encuentran asegurados -según la estrategia mencionada- por una serie de prioridades estratégicas nacionales: la defensa nacional, la seguridad estatal y pública, el crecimiento económico, el desarrollo tecnológico y la educación, la salud pública, la cultura, el uso racional de los recursos naturales y la estabilidad estratégica junto a las asociaciones estratégicas igualitarias. Los lineamientos planteados en sus principales documentos oficiales y la práctica externa de sus asuntos estratégicos apoyan una aproximación pragmática cuya trayectoria se adapta a los cambios en el entorno regional y global.

Una vez dilucidada la orientación -o lineamientos generales para la acción-, es crucial hacer hincapié en los objetivos de la estrategia. En términos generales, Rusia ha planteado

${ }^{21}$ Russian Federation Presidency, Russian Federation's National Security Strategy, Moscú, Kremlin, Moscow, 2015.

${ }^{22}$ Russian Federation Presidency, The Foreign Policy Concept of the Russian Federation, Moscú, Kremlin, 2016. 
tres objetivos permanentes a lo largo de las últimas dos décadas: asegurar la primacía rusa en el espacio postsoviético, preservar el estatus de gran potencia dentro de un mundo policéntrico y disuadir la amenaza que representa la expansión de la otan sobre su espacio de influencia. En el discurso de Vladimir Putin en la Conferencia sobre Seguridad de Múnich, en 2007, expresó:

Creo que es obvio que la expansión de la OTAN no tiene ninguna relación con la modernización de la propia Alianza o con la garantía de seguridad en Europa. Por el contrario, representa una grave provocación que reduce el nivel de confianza mutua. Y tenemos el derecho de preguntar: ¿contra quién está destinada esta expansión?. ${ }^{23}$

Dado que la orientación no se encuentra claramente definida, sino que opera pragmáticamente en un contexto de gran incertidumbre externa para sostener su rol como potencia euroasiática, Moscú propuso una narrativa multipolar favorable a sus intereses. En ésta, el mundo se presenta como un mundo policéntrico centrado en tres bloques: euroatlántico, euroasiático y Asia-Pacífico, liderados respectivamente por EEuU, la Federación de Rusia y la República Popular China. ${ }^{24}$ En este sentido, el orden internacional no es hegemónico sino multipolar, crecientemente democrático y postoccidental. ${ }^{25}$ La afirmación sobre esta nueva trayectoria del sistema

${ }^{23}$ Vladimir Putin, "Speech and the Following Discussion at the Munich Conference on Security Policy", Múnich, Kremlin, 2007.

${ }^{24}$ Expresado en Russian Federation Presidency, The Foreign Policy Concept... op. cit., en varios años: 2008, 2013, 2016.

${ }^{25}$ En el diseño de la política exterior rusa, América Latina y el Caribe se presenta como un socio importante para el mundo multipolar además de una región relevante para medir su grado de influencia como actor global. Sin embargo, su lugar en la agenda internacional rusa todavía es secundario y periférico. Para más detalles sobre las relaciones entre Rusia y América Latina ver Victor Jeifets, "Russia is coming back to Latin America: perspectives and obstacles", Anuario de Integración 11, 2015; Vladimir Rouvinski, "Understanding Russian Priorities in Latin America", 
internacional no es solamente una descripción, sino una afirmación propositiva en la cual Rusia tiene un rol fundamental como gran potencia.

En la Estrategia de Seguridad Nacional 2015 se afirma que el "proceso de conformar un nuevo modelo policéntrico del orden mundial está siendo acompañado por un aumento en la inestabilidad global y regional", que en la implementación de una política exterior e interior independiente de Rusia ha generado, como ya se mencionó previamente, "la oposición de EEUU y sus aliados, que buscan mantener su dominio en los asuntos mundiales. Entre las políticas implementadas de contención a Rusia, se contemplan el ejercicio de presiones políticas, económicas, militares e informativas sobre ella”. ${ }^{26}$ De una manera más ofensiva, Leonid Ivashov, el geopolítico experto en la región euroasiática, afirma que "la principal fuente de la amenaza geopolítica para Rusia es la comunidad transnacional y la civilización occidental, principalmente EEUU, que reclaman la hegemonía mundial con la eliminación de todos los centros alternativos de poder en un mundo unipolar". ${ }^{27}$ En una visión crítica, Kissinger afirma que la concepción distintiva del orden internacional ruso que trata de lograr una seguridad absoluta genera inseguridad absoluta en muchos de sus vecinos. ${ }^{28}$

Kennan Cable 20, 2017 y Ariel González Levaggi, "Latin America faces Eurasian conflicts: assessing regional responses in the age of Russia-United States tensions", Vestnik St Petersburg University, International Relations 12 (2), pp.198-209.

26 Russian Federation Presidency, Russian Federation's National..., op. cit.

27 Leonid Ivashov, “Состояние системы международной безопасности на современном этапе” (El estado del sistema de seguridad internacional en la etapa actual). Известия Уральского федерального университета (Revista de la Universidad Federal de los Urales), Serie 3, Ciencias Sociales 8, 3 (2013), pp. 6-18.

28 Henry Kissinger, "Chaos and order in a changing world", CAPX, https:/ / capx.co/chaos-and-order-in-a-changing-world/ 
Instrumentos: diplomacia, soft power y (bastante) coerción

En línea con sus objetivos primariamente geopolíticos, la gran estrategia rusa se caracteriza por el uso de la coerción mediante la utilización de amenazas diplomáticas, la instrumentalización estratégica de los recursos energéticos, la interferencia electoral o inclusive la aplicación directa del factor militar. En este contexto, tres elementos son cruciales para la implementación de su estrategia internacional: diplomacia tradicional, el poder suave con elementos punzantes y el uso de la fuerza.

Con un cuerpo altamente profesional y experimentado, la diplomacia rusa tiene una proyección con alcance global. El Ministerio de Asuntos Exteriores cuenta con 242 puestos diplomáticos alrededor del mundo. Con esas capacidades, Rusia se ubica en el $4^{\text {to }}$ lugar del Índice de Diplomacia Global del Instituto Lowy ${ }^{29}$ y el $7^{\text {mo }}$ del Índice Elcano de Presencia Global con una preminencia del factor militar sobre el económico y "suave". ${ }^{30}$ De modo complementario, Rusia ha desarrollado una serie de instituciones que le permite crear estrategias de proyección no tradicional más allá de sus instrumentos tradicionales, las Fuerzas Militares y de Seguridad junto al Ministerio de Asuntos Exteriores. El complejo de poder suave ruso incluye desde la promoción de la cultura, becas y lengua rusa por la Agencia Federal para los Asuntos de la Comunidad de Estados Independientes, los Compatriotas en el Extranjero y Cooperación Humanitaria Internacional (bajo jurisdicción del Ministerio de Asuntos Exteriores) y la Fundación Russkiy Mir, hasta el desarrollo de una plataforma global de información con la cadena audiovisual RT y la agencia de noticias Sputnik. En el caso de los instrumentos mediáticos, la fuerte conexión entre los medios globales rusos y el

29 Globlal Diplomacy Index, https://www.lowyinstitute.org/globaldiplomacy-index-introduction

30 Índice Elcano de Presencia Global, https://www.globalpresence. realinstitutoelcano.org/es/ 
mensaje oficial del Kremlin borra las líneas entre la información y la propaganda. De este modo, si bien han permitido desarrollar un alcance informativo global, su efectividad -medida en términos de audiencia y recepción de los mensajes- dista de ser óptima. Además, en una zona gris entre lo informativo y lo coercitivo se desarrollan acciones punzantes. En una crítica a las conductas internacionales de Rusia y China, Walker incorpora el concepto de sharp power (poder punzante) por el cual la implementación de acciones de manipulación informativa mina la integridad de las instituciones democráticas, por ejemplo, en el caso de elecciones. ${ }^{31} \mathrm{Si}$ bien Moscú ha negado cualquier interferencia en procesos electorales y el alcance de sus herramientas parecería no tener un carácter tan efectivo en entornos occidentales, dicha aproximación sí tiene mayor influencia en su vecindario, donde posee redes asentadas en un legado cultural, político y económico.

Finalmente, Moscú posee una superioridad militar en el espacio euroasiático, tanto en sus capacidades como en la exportación de material bélico. En lo que respecta a la doctrina, la Federación de Rusia abandonó el modelo de disuasión nuclear y guerra convencional para adoptar una confrontación híbrida en la cual los límites entre los instrumentos militares y no-militares son borrosos, así como la distinción entre las situaciones de conflicto armado y la paz. ${ }^{32}$ Además, luego de la guerra ruso-georgiana en 2008, las Fuerzas Armadas imple-

${ }^{31}$ Christopher Walker, "What is Sharp Power?", Journal of Democracy 29, núm. 3 (2018), pp. 11-12.

${ }^{32} \mathrm{El}$ caso más patente fue la crisis de Ucrania a partir 2014. Una de las cuestiones más polémicas sobre la estrategia militar rusa es la identificación de la guerra híbrida como el principal eje de acción operativo. La concepción de guerra híbrida fue desarrollada originalmente en EEUU por William Nemeth y luego desarrollada por Frank Hoffman. Sin embargo, en concepto más utilizado en Rusia es el de "guerra no lineal", incorporado por el jefe de Estado Mayor, Valery Gerasimov, como la principal característica de la guerra contemporánea marcada por la importancia de los métodos "indirectos y asimétricos". Véase C. Bartles, "Getting Gerasimov Right", Military Review, enero-febrero de 2016, p. 33-35. 
mentaron un programa de modernización con un programa de armamento estatal centrado en la incorporación de material avanzado para los diferentes servicios. La Fuerza Aérea incorporó aviones de caza Su-30SM, Su-35 y Su-34 y helicópteros Mi-28N, Mi-35 y Ka-52; el ejército mejoró sus medios en materia de tanques de batalla principales, vehículos de combate de infantería y transportes blindados de personal, mientras la Armada modernizó su flota submarina y transformó el perfil de su flota de superficie con la incorporación de buques multipropósito, con capacidades antisuperficie y ataque terrestre que incorporan misiles crucero. Otros desarrollos interesantes se dieron en sistemas de defensa antiaéreo (S-400), unidades de comunicación, municiones guiadas y sistemas de guerra electrónica, entre otros. ${ }^{33}$ En términos generales, los resultados han sido bastante promisorios con las operaciones "no lineales" de Crimea, en 2014, y la proyección estratégica en el conflicto sirio a partir de 2015.

\section{El péndulo: entre Eurasia y Occidente}

La reconstrucción de la agencia internacional de la Federación de Rusia llegó a su clímax con la intervención militar en el conflicto sirio. Desde la llegada de Vladimir Putin la política exterior y de seguridad internacional de Moscú pasó por una serie de etapas marcadas por el progresivo alejamiento de Occidente y la recuperación de la centralidad geopolítica en el espacio euroasiático. De manera simultánea, la mayor distancia con respecto a EEUU y sus socios europeos reportó una modificación en la percepción de amenazas por el nodo ejecutivo, al trasladar las preocupaciones desde una plataforma de amenazas comunes ha-

${ }^{33}$ Michael Kofman, "The Russian Military: A Force in Transition”, en Alexander Golts y Michael Kofman (eds.), Russia's Military Assessment, Strategy, and Threat, Washington, DC, Center on Global Interests, 2016, pp. 4-7. 
cia la identificación de las políticas de expansión de la Unión Europea y la OTAN como una amenaza a su existencia.

El eje de la política internacional rusa en la post Guerra Fría ha sufrido una lenta traslación desde del eje euroatlántico $^{34}$ al euroasiático y Pacífico. ${ }^{35} \mathrm{Si}$ bien el vector occidental sigue siendo el principal estructurador de la proyección internacional rusa, en los últimos años ha crecido el peso del vector eurasiático, que ofrece una visión más equilibrada sobre la inserción global de la Federación de Rusia en el marco de un sistema internacional marcado por la incertidumbre y la creciente multipolaridad. En este sentido, un factor clave ha sido la proyección económica y política de Beijing en su vecindario noroccidental junto con una creciente convergencia de agendas con Moscú, que incluye importantes inversiones en el campo energético -se destaca el acuerdo del gaseoducto "Poder de Siberia" por treinta años-, el swap (intercambio) de monedas para reemplazar al dólar como moneda de cambio comercial, además de convergencias institucionales en el plano regional con la profundización de la Organización de Cooperación de Shanghái, la incorporación de Moscú al proyecto chino de la Iniciativa del Cinturón y la Ruta de la Seda, y la convergencia de posiciones en el marco del Consejo de Seguridad de la Organización de las Naciones Unidas (ONU).

En esta línea, las relaciones entre Rusia y Occidente son el eje que han condicionado y estructurado la agenda global rusa, por lo menos hasta el ascenso de la República Popular China, que comenzó a influir seriamente en el cálculo del Kremlin desde fines del año 2000 con la estructuración del grupo BRICS y el fortalecimiento del vector euroasiático de su política exterior. Las etapas que definen la política exterior en la era Putin en relación con Occidente son de aproximación tímida (2000-2003), competencia limitada (2003-2013) y desconfianza generalizada (2014-).

34 Jeffrey Mankoff, Russian Foreign Policy: The Return of Great Power Politics, Nueva York, Rowman \& Littlefield, 2011.

35 Trenin, art. cit. 
$\mathrm{Al}$ asumir como presidente, Putin hereda la aproximación 'pro-occidental' de su antecesor Boris Yeltsin, ${ }^{36}$ aunque las relaciones se encontraban en una situación frágil debido a la expansión de la OTAN. Una de las razones que explican el fortalecimiento de los sectores más conservadores dentro del Kremlin y el desplazamiento de los actores pro-occidentales en favor de nacionalistas estatistas y eurasianistas tiene un grado de correlación elevado con el escenario internacional de la Post Guerra Fría, especialmente a partir del desencanto de la elite política rusa con la fallida visión paneuropeísta y la ampliación de la OTAN en la zona tradicional de influencia rusa o, como la denomina la doctrina rusa, el "exterior cercano". 37

La ruptura de la incipiente asociación ruso-estadounidense en el periodo inmediatamente posterior al fin de la Guerra Fría ha dado lugar a diferentes interpretaciones. Desde la perspectiva estadounidense, en general se le asigna responsabilidad al rol "autocrático" y "neoimperialista" de Vladimir Putin, mientras que una perspectiva más crítica enfatiza la explotación de la debilidad rusa por las políticas de EEUU, mediante el crecimiento de un cerco militar con bases e infraestructura de la OTAN, reiteradas intervenciones en los asuntos internos rusos, la negación de las compromisos de seguridad regional en las fronteras de Rusia y los intentos de alcanzar la supremacía nuclear, que neutraliza las capacidades de su tradicional rival. ${ }^{38}$

Si bien durante los años noventa hubo tensiones en diversos temas de la agenda, como las operaciones de la OTAN en los Balcanes, el principal motivo de la divergencia de visiones con EeUU fue la decisión de Washington de expandir

36 Mariano Caucino, La Rusia de Putin, Buenos Aires, Ediciones B, 2016, p. 71.

${ }^{37}$ Robert Donaldson y Joseph Nogee, The foreign policy of Russia: Changing systems, enduring interests, Armonk, Nueva York, M.E. Sharpe, 2009, p. 163 .

${ }^{38}$ Stephen Cohen, Soviet Fates and Lost Alternatives: From Stalinism to the New Cold War, Nueva York, Columbia University Press, 2009, pp. 166-170. 
la membresía de la oTAN. ${ }^{39}$ Esta expansión fue el "pecado original" que convenció al liderazgo ruso de que la política de EEUU hacia Moscú no reflejaba una "asociación estratégica" sino una búsqueda de dominación. ${ }^{40} \mathrm{~A}$ partir de la transformación del Concepto Estratégico de la OTAN, aprobado en la Cumbre de Washington de 1999, se fomentó la expansión hacia el este con la incorporación de la República Checa, Hungría y Polonia, además de la aprobación del Mecanismo de Plan de Acción para nuevas incorporaciones. En los años posteriores, la OTAN se ampliaría al resto de los países de Europea del Este, así como países que habían pertenecido a la URSs, tales como Estonia, Letonia y Lituania, lo que generó mayores incentivos para la estructuración de un orden regional confrontativo en Eurasia Central. ${ }^{41}$ La expansión occidental comenzó a ser la principal preocupación de la dirigencia rusa que, con el despliegue de sistemas de defensa estratégica en los países del este europeo, comenzará a ser percibida como la principal amenaza a su seguridad nacional. ${ }^{42}$

Además de la percepción de amenaza hacia Occidente, hay una serie de factores históricos que inciden en la agenda internacional rusa, como la existencia de fronteras permeables a lo largo de la periferia territorial, un Estado y sociedad multicultural compuesta por bloques etnoterritoriales y el

39 Alexei Pushkov, “Don't Isolate Us: a Russian View of NATO Expansion”, National Interest 47 (1997), p. 58.

${ }^{40}$ Cohen, op. cit., p. 189

41 Ariel González Levaggi, Confrontational and cooperative regional orders: managing regional security in world politics, Londres, Routledge, 2020, p. 45 .

${ }^{42}$ La doctrina militar 2010 presenta como peligro externo primario el "deseo de dotar al potencial de la fuerza de la Organización del Tratado del Atlántico Norte (OTAN) con funciones globales desarrolladas en violación de las normas del derecho internacional y para desplazar infraestructura militar de los países miembros de la OTAN a las fronteras de la Federación de Rusia, incluso mediante la expansión del bloque", en Russian Federation Presidency, Military Doctrine of the Russian Federation, Moscú, Kremlin, 2010. 
atraso económico comparado con los países desarrollados. ${ }^{43}$ Desde el punto de vista de la seguridad del Estado, las primeras dos cuestiones son críticas. En primer lugar, la percepción de inseguridad e incertidumbre sobre fronteras de un territorio inmenso, especialmente si involucran alianzas militares con despliegue de infraestructura militar avanzada, que bloquearía los sistemas ofensivos convencionales y no-convencionales rusos. En segundo lugar, la existencia de bloques etnonacionales presenta un desafío interno debido a potenciales intentos de separatismo, amenazas de uso de la violencia terrorista y la expansión de ideologías fundamentalistas.

Vinculado al segundo punto, los ataques terroristas del $11 \mathrm{~S}$ permitieron desarrollar una cooperación concreta en dos campos de la seguridad internacional, la lucha contra el terrorismo y la colaboración logística en la campaña militar contra los Talibán en Afganistán, que incluyó el visto bueno para el despliegue de fuerzas estadounidenses en Kirguistán y Uzbekistán. En el marco de la guerra contra el terrorismo, se sucedieron una serie de señales positivas, como el cierre de las bases de inteligencia en Cuba (Lourdes) y naval en Vietnam (Cam Ranh Bay), mientras que EeuU clasificó a Rusia como una economía de mercado, lo que abrió la puerta para su ingreso en la Organización Mundial del Comercio (oMC).

Sin embargo, la herencia no conllevó de manera automática la continuación de un rapprochement ruso-norteamericano, que había pasado su mejor momento a principios de los años noventa. Cambios producidos a nivel internacional y domésticos, tanto en Moscú como en Washington, pusieron a los dos titanes de la Guerra Fría nuevamente en situación de colisión. La estrategia de los "halcones neoconservadores" en Medio Oriente con los planes de invasión de Iraq enturbiaron las relaciones bilaterales, dada la oposición a una in-

${ }^{43}$ Alfred Rieber, "Persistent factors in Russian foreign policy: an interpretative essay", en Hugh Ragsdale (ed.), Imperial Russian foreign policy, Cambridge, University Press, 1993, p. 315-359. 
tervención armada y el posterior veto ruso en el Consejo de Seguridad de Naciones Unidas. Al mismo tiempo, la reactivación de los planes de expansión de la OTAN hacia Europa del Este y los países bálticos terminó de modificar la percepción del Kremlin sobre Eeuu, ya que el soporte a la "Nueva Europa" -frase popularizada por el secretario de Defensa Donald Rumsfeld-, aliada en el esfuerzo de guerra en Iraq, también se ponía en tensión con la estrategia de seguridad regional de Moscú en su frontera occidental.

La incorporación de Bulgaria, Rumania, Estonia, Lituania, Letonia, Eslovaquia y Eslovenia en 2004 -que además se incorporaron a la Unión Europea el mismo año, salvo los dos primeros casos- junto a la invasión de Iraq afectaron seriamente el diálogo estratégico. A partir de ese momento, las relaciones entrarán en un espiral de antagonismo con intentos fallidos de normalizar los vínculos a fines del año 2000. Luego de la expansión en el este europeo, Robert Gates, entonces secretario de Defensa, recomendó la continuación del Programa de Defensa Misilística Estratégica en Europa Oriental, lo que despertó críticas en Moscú por la ubicación de infraestructura militar crítica cerca de sus fronteras.

En el marco del deterioro de las relaciones, el clímax será el conflicto ruso-georgiano (2008) en el cual Moscú realizó una exitosa demostración de fuerza para señalar los límites de las actividades de un país percibido como proxy de Occidente. Las aspiraciones georgianas para incorporarse a la constelación occidental y las señales ambiguas de Washington y Bruselas generaron una reacción rusa que fomentó unilateralmente la secesión de Abjazia y Osetia del Sur, luego del conflicto armado.

Con la llegada de Barack Obama a la presidencia en EEUU, se intentó una nueva aproximación con la política del "reseteo", que planteó el congelamiento de los proyectos de expansión misilística y la firma de un nuevo tratado START. La iniciativa se frustró con las declaraciones de la diplomacia estadounidense durante las protestas en Moscú de 2011 y las posteriores reacciones rusas. Además, la continuación del 
Programa de Defensa Misilística influyó también para que el tercer periodo de Vladimir Putin en la presidencia tuviera un carácter más ríspido en relación con Occidente. Aunado a esto, tres temas de la agenda regional y global pusieron los intereses rusos en conflicto con Occidente. En primer lugar, las excesivas operaciones de la OTAN en Libia, que llevaron al derrocamiento de Gadafi, frustraron la posición rusa. Durante el periodo del primer ministro Medvédev, Moscú había habilitado la aprobación de la resolución en el Consejo de Seguridad de Naciones para la implementación de una "zona de no vuelo". En segundo lugar, la desestabilización de la Siria de Al-Assad -su principal aliado en Medio Oriente- por países aliados a EEUU, junto con la intención para intervenir militarmente de un sector de la OTAN ante una serie de supuestos ataques con armas químicas del gobierno sirio a la oposición. Por último, la expansión de las instituciones occidentales en el espacio postsoviético, particularmente en Ucrania.

El caso ucraniano profundizó la fractura ruso-occidental. Luego de un fallido intento de firma del Acuerdo de Asociación entre Ucrania y la Unión Europea debido a presiones rusas estalló la "revolución de Euromaidán". Una serie de movilizaciones favorables a la integración al espacio europeo protestó contra la suspensión del acuerdo y forzó la resignación de Víktor Yanukóvich, cuyo capital político se concentraba en las zonas más afines a Rusia, Crimea y las provincias orientales. Sin embargo, el conflicto se internacionalizó rápidamente. EeUU y la Unión Europea explícitamente apoyaron un cambio político en Kiev, mientras Moscú se opuso. Ante la retirada obligada de Yanukóvich, Putin tomó la decisión de anexar Crimea y apoyar a los rebeldes de las provincias de Donetsk y Luhansk. La respuesta de Occidente fue inmediata. Tanto Washington como Bruselas impusieron duras sanciones tanto a los involucrados en la crisis ucraniana, y no solamente individuos, sino también empresas en diferentes sectores. 
Siguiendo la lógica de acción iniciada en Georgia, Rusia aplicó un neorrevisionismo que, si bien no ha buscado cambiar los principios fundamentales del orden internacional, trata de asegurar que los intereses rusos sean respetados en el área de su incumbencia. ${ }^{44}$ Sin embargo, dicha conducta implica afectar de manera directa el principio de soberanía de terceros países, como es el caso de la anexión de Crimea o el establecimiento y sostenimiento de pseudo Estados como Osetia del Sur y Abjasia. Dado el enfrentamiento normativo y estratégico que trae aparejada esta situación, la política internacional rusa mutó a "modo de guerra":

El objetivo inmediato de la política exterior de Moscú es resistir la presión que le imponen Washington y sus aliados [...] el Kremlin ha estado operando de facto en un modo de guerra, y el presidente ruso, Vladimir Putin, ha estado actuando como un líder de la guerra. Hasta ahora, el Kremlin se ha mantenido firme, sin ceder terreno a sus oponentes. ${ }^{45}$

Además de lidiar con las sanciones, Rusia comenzó a involucrarse con mayor profundidad en espacios no tradicionales, tanto para apoyar a sus aliados como para ocupar aquellos espacios "abandonados" por EeuU en el marco de la retracción del gran Medio Oriente, impulsada por la administración Obama y continuada en la era Trump. Una de las pruebas críticas fue el caso de Siria, donde la Federación desplegó -con el visto bueno del gobierno de Al-Assad- una operación aeronaval y terrestre de importantes magnitudes que le permitió cambiar el rumbo del conflicto interno sirio y colaborar con la derrota del Estado Islámico. Por otro lado, comenzó a explorar el eje asiático de su aproximación exterior mediante el acercamiento progresivo con China, asentado

${ }^{44}$ Richard Sakwa, Frontline Ukraine: Crisis in the Borderlands, Londres, I.B. Tauris, 2015, p. 7.

${ }^{45}$ Dmitri Trenin, "Demands on Russian foreign policy and its drivers: Looking out five years", Moscow Carnegie Center, 2017, https://carnegie.ru/commentary/72799 
en múltiples proyectos de cooperación económica y energética, además de continuar la tradicional cooperación en el área militar. En este contexto, la República Popular China aparece como un eje compensador del tradicional peso de Occidente en la proyección internacional de Moscú.

Ante la implosión de la uRss, la Federación de Rusia encaró una política exterior "normal" frente a la inexistencia de una rivalidad ideológica y la necesidad de lidiar con las estrategias internacionales de los países postsoviéticos. ${ }^{46} \mathrm{Si}$ en el epílogo de la Unión Soviética y los primeros años de la presidencia de Boris Yeltsin había expectativa real sobre una nueva etapa de relaciones con EEUU en particular, o con Occidente en general, sobre la base de una visión de una "Gran Europa" que generaba expectativas de incorporación de la Federación de Rusia a las instituciones occidentales:

Occidente invitó a Rusia a unirse a una Comunidad Atlántica ampliada, pero Rusia buscó unirse a un Occidente transformado y a una Europa reconfigurada, objetivos que siguen activos hasta el día de hoy. En otras palabras, a Rusia se le ofreció ser miembro del Occidente histórico, pero unirse habría implicado cambiar algunos elementos fundamentales de su identidad histórica. En cambio, Rusia asumió que el premio de la membresía rusa habría tenido tal importancia de época que el Occidente histórico se transformaría fácilmente en otra cosa, que en opinión de Moscú se habría convertido en un Gran Occidente. ${ }^{47}$

El deterioro de las relaciones con Occidente tensionó la política internacional de Rusia en direcciones que generaron situaciones críticas que profundizaron la distancia. Ni el fin de la era Obama ni la llegada de Donald Trump facilitaron las relaciones bilaterales, ni colaboraron con la

46 Nikolas Gvosdev y Christopher Marsh, Russian Foreign Policy: Interests, Vectors, and Sectors, Washington, CQ Press, 2013, pp. 4-5.

${ }^{47}$ Richard Sakwa, Russia against the rest: The post-Cold War crisis of world order, Cambridge, University Press, 2017, p. 13. 
atenuación de las sanciones. La desconfianza generalizada ha permeado la percepción de las burocracias en ambos lados del Pacífico, más allá de los intentos de los actuales líderes para establecer un nuevo "reseteo", como se demostró en el encuentro de Helsinki entre Putin y Trump en julio de 2018. Además de formalmente colocar a Rusia como "competidor estratégico", en la Estrategia de Seguridad Nacional de 2018, Washington denunció el Tratado de Fuerzas Nucleares de Rango Intermedio (INF) - con el argumento de que Moscú había incumplido sus compromisos-, mientras que la crisis en Ucrania sigue latente y los intereses divergentes en torno a Siria e Irán agregan mayor tensión, sin horizonte de resolución para lograr las bases de una región euroasiática más estable.

En este contexto, la transición del eje estructurante de la acción internacional de Rusia presenta tres consecuencias para el actual orden internacional. En primer lugar, el uso de la fuerza militar ha desgastado la legitimidad de los principios rectores del sistema de posguerra. En segundo lugar, si bien la agencia rusa en el sistema internacional ha visto un notable crecimiento, la conducta asertiva afectó tanto la capacidad económica -debido a las sanciones internacionales- como la atracción de su narrativa. Por último, el "eje euroasiático" entre Moscú y Beijing, cuyo acercamiento parece reflejar una "alianza sólida y profundamente arraigada", 48 ha comenzado a alterar los fundamentos del mundo de post Guerra Fría.

\section{Conclusiones}

Rusia regresó a las grandes ligas de la política global. Los tres elementos constitutivos de una gran estrategia -su principio ordenador, los objetivos y sus instrumentos- nos permiten dar con ciertas claves para interpretar la conducta rusa en la

48 Andrés Serbin, Eurasia y América Latina en un mundo multipolar, Buenos Aires, CRIES y Editorial Icaria, 2019, p. 72. 
era Putin. Si bien el declive del orden unipolar estadounidense facilitó el fortalecimiento en el espacio euroasiático, el reposicionamiento global ruso se explica además por dos factores domésticos: la centralización política bajo Vladimir Putin, que permitió una continuidad en el plano institucional, y la capacidad burocrática para lograr un consenso sobre asuntos estratégicos. El resultado fue el desarrollo de una gran estrategia pragmática enfocada en mantener el estatus de gran potencia a nivel internacional, limitar la expansión de la infraestructura política y militar OTAN en su frontera occidental y sostener su posición hegemónica en el espacio postsoviético.

En este sentido, el trípode de objetivos tensionó la relación las principales potencias occidentales, tanto por la aspiración de reconstruir el liderazgo en el espacio postsoviético como por la intención de bloquear la expansión de la OTAN, al mismo tiempo que acercó posiciones con potencias no occidentales, tanto en el espacio euroasiático como en otras regiones, articulado por medio del foro BRICS. Las constantes crisis en el vecindario ruso no eximen a Moscú de responsabilidad, a pesar de la presencia de las incisivas acciones de EEUU y sus aliados de la otan. De todas maneras, la permanencia de las tensiones ruso-estadounidenses ha tenido un efecto importante en la orientación de la estrategia internacional rusa. Moscú ha trasladado el centro de sus acciones desde el eje occidental hacia el espacio euroasiático, priorizando el espacio postsoviético, desarrollando un inusitado activismo en Medio Oriente y acercándose a la República Popular China como un factor de contrapeso frente a las presiones y sanciones occidentales.

La perspectiva de realismo neoclásico utilizada en el artículo permite integrar factores domésticos en los procesos de transformación global, además de proveer un marco explicativo sobre la conducta internacional de los Estados, en el cual priman los temas de alta política. Sin embargo, para ampliar el campo de análisis de la política exterior rusa es importante incorporar marcos analíticos complementarios, 
como los abordajes de geopolítica neoclásica, la literatura constructivista sobre la identidad nacional rusa, estudios sobre el rol de Rusia en la política económica internacional, además de estudios de caso que evalúen la efectividad (o no) de la implementación de la estrategia general rusa a nivel de política regional y bilateral, así como la evolución de las relaciones bilaterales con EeuU y la República Popular China.

\section{BibLIOGRAFÍA}

Caucino, Mariano, La Rusia de Putin, Buenos Aires, Ediciones B, 2016.

Chaguaceda, Armando, "The Putin System: Russian Authoritarianism Today", Revista Mexicana de Análisis Político y Administración Publica, 2016, 5 (1), pp.75-92.

Cohen, Stephen, Soviet Fates and Lost Alternatives: From Stalinism to the New Cold War, Nueva York, Columbia University Press, 2009.

Cohen, Stephen, Why Cold War Again? How America Lost Post-Soviet Russia, Londres, I.B. Tauris, 2017.

Donaldson, Robert y Joseph Nogee, The foreign policy of Russia: Changing systems, enduring interests, Armonk, NY, M.E. Sharpe, 2009.

Gelman, Vladimir, Authoritarian Russia. Analyzing Post-Soviet Regime Changes, Pensilvania, Pittsburg University Press, 2015.

Gleb, Pavlovsky, "Russian Politics under Putin”, Foreign Affairs, mayo-junio de 2016.

Gvosdev, Nikolas y Christopher Marsh, Russian Foreign Policy: Interests, Vectors, and Sectors, Washington, CQ Press, 2013.

Ivashov, Leonid, "Sostoianie sistemy mezhdunarodnoi bezopasnosti na sovremennom etape" (E1 Estado del sistema de seguridad internacional en la etapa actual), Известия Уральского федерального университета (Revista de la Universidad Federal de los Urales), Serie 3, Ciencias Sociales 8 (3), 2013, pp. 6-18.

JeIfets, Victor, "Russia is coming back to Latin America: perspectives and obstacles", Anuario de Integración 11, 2015. 
Kennedy, Paul, "Grand Strategy in War and Peace: Toward a Broader Definition", en Paul Kennedy (ed.), Grand Strategies in War and Peace, New Haven, Yale University Press, 1991, pp. 1-7.

Kissinger, Henry, "Chaos and order in a changing world", CAPX, 2017, https://capx.co/chaos-and-order-in-a-changing-world/ Kofman , Michael, "Raiding and International Brigandry: Russia's Strategy for Great Power Competition”, War on the Rocks, 2018, https:/ / warontherocks.com/2018/06/raiding-and-international-brigandry-russias-strategy-for-great-power-competition/

Kofman, Michael, "Russian defense spending is much larger, and more sustainable than it seems", Defense News, 2019, https:/ / www.defensenews.com/opinion/commentary/2019/05/03/ russian-defense-spending-is-much-larger-and-more-sustainable-than-it-seems /

Legvold, Robert, Return to Cold War, Londres, Polity, 2016.

Lucas, Edward, The New Cold War: The Future of Russia and the Threat to the West, Nueva York, Palgrave Macmillan, 2008.

Mankoff, Jeffrey, Russian Foreign Policy: The Return of Great Power Politics, Nueva York, Rowman \& Littlefield, 2011.

McFaul, Michael, From Cold War to Hot Peace. An American Ambassador in Putin's Russia, Nueva York, Houghton Mifflin Harcourt, 2018.

Mijares, Víctor M., "Realismo neoclásico: ¿El retorno de los estudios internacionales a la ciencia política?", Revista de Ciencia Política (Santiago), 2015, 35(3), pp. 581-603.

Minchenko, Evgeny, "Politburo 2.0: Renovation instead of dismantling", Minchenko Consulting Communications Group, 2017.

Monaghan, Andrew, "Defibrillating the Vertical? Putin and Russian Grand Strategy", Research Paper, Londres, Chatham House, 2014.

Neumann, Iver, Russia and the Idea of Europe. A Study in Identity and International Relations, Nueva York, Routledge, 1996.

Pushkov, Alexei, "Don't Isolate Us: a Russian View of NATO Expansion”, National Interest 47, 1997, pp. 58-63.

Putin, Vladimir, "Speech and the Following Discussion at the Munich Conference on Security Policy”, Múnich, Kremlin, 2007. 
Rieber, Alfred, "Persistent factors in Russian foreign policy: an interpretative essay", en Hugh Ragsdale (ed.), Imperial Russian foreign policy, Cambridge, University Press, 1993, pp. 315-359.

Rouvinski, Vladimir, "Understanding Russian Priorities in Latin America”, Kennan Cable 20, 2017.

Russian Federation Presidency, Military Doctrine of the Russian Federation, Moscú, Kremlin, 2010.

Russian Federation Presidency, Russian Federation's National Security Strategy, Moscú, Kremlin, 2015.

Russian Federation Presidency, The Foreign Policy Concept of the Russian Federation, Moscú, Kremlin, 2008, 2013, 2016.

SAKwa, Richard, "Dualism at Home and Abroad: Russian Foreign Policy Neo-Revisionism and Bicontinentalism", en David Cadier y Margot Light (eds.), Russia's Foreign Policy, London, Palgrave Macmillan, 2015, pp. 65-79.

SAKwa, Richard. Frontline Ukraine: Crisis in the Borderlands, Londres, I.B. Tauris, 2015.

SAKWA, Richard, Russia against the rest: The post-Cold War crisis of world order, Cambridge, University Press, 2017.

Schoen, Douglas y Evan Smith, Putin's Master Plan: To Destroy Europe, Divide NATO, and Restore Russian Power and Global Influence, Nueva York, Encounter Books, 2016.

Sserbin, Andrés, Eurasia y América Latina en un mundo multipolar, Buenos Aires, CRIES y Editorial Icaria. 2019.

Taliaferro, Jeffrey, Norrin Ripsman y Steven Lobell, "Introduction: Grand Strategy between the World Wars", en Jeffrey Taliaferro, Norrin Ripsman y Steven Lobell (eds.), The Challenge of Grand Strategy: The Great Powers and the Broken Balance between the World Wars, Nueva York, Cambridge University Press, 2012, pp. 1-36.

Trenin, Dmitri, "Demands on Russian foreign policy and its drivers: Looking out five years", Moscow Carnegie Center, 2017, https://carnegie.ru/commentary/72799

Trenin, Dmitri, "Vladimir Putin's Fourth Vector, Russia in Global Affairs", Russia in Global Affairs, enero-marzo de 2018, https:/ / eng.globalaffairs.ru/number/Vladimir-Putins-Fourth-Vector19441 
Tsygankov, Andrei, "Preserving Influence in a Changing World:

Russia's Grand Strategy", Problems of Post-Communism, núm. 58 (2), 2011, pp. 28-44.

Tsygankov, Andrei, Russia's Foreign Policy: Change and Continuity in National Identity, Lanham, MD, Rowman \& Littlefield, 2016.

Tsygankov, Andrei, Russia and the West from Alexander to Putin: Honor in international relations, Cambridge, University Press, 2012. Walker, Christopher, "What is Sharp Power?", Journal of Democracy 29 (3), 2018, pp. 9-23.

Wallander, Celeste, "Russia: The Domestic Sources of a Less-thana-Grand Strategy", en Ashley J. Tellis y Michael Wills (eds.), Strategic Asia 2007-2008: Domestic Political Change and Grand Strategy, Seattle, National Bureau of Asian Research, 2007. 